\title{
Two Cases of Leiomyosarcoma of the Duodenum
}

\section{T. E. STARZL, Ph.D., M.D.; VICTOR M. BernhaRd, M.D., and GEORGe C. HeNeger, M.D., Chicago}

Since the first report of leiomyosarcoma of the duodenum in 1920, approximately 60 cases have been described. ${ }^{5}$ The number of patients in whom the tumor was successfully removed has, however, been small. Many of the reported cases were first diagnosed at autopsy, and others were nonresectable at the time of operation. In addition, the mortality rate in the early attempts at resection was high, being approximately $70 \%$ in the collected series of Weinstein and Roberts ${ }^{6}$ in 1953.

The purpose of the present report is to document two additional examples of successfully treated leiomyosarcoma of the duodenum. In one patient a tumor in the fourth part of the duodenum was removed with segmental resection. The neoplasm in the other patient was in the second part of the duodenum, necessitating pancreaticoduodenal resection.

\section{Report of Cases}

CASE 1 (Veterans Administration Hospital, Chicago).-This 42-year-old white man was admitted to the hospital after 10 months of recurrent major and minor gastrointestinal bleeding. During two previous admissions to other hospitals, complete gastrointestinal work-ups, including esophagoscopy and gastroscopy, had been negative. He hard never noted abdominal pain, nausea, vomiting, constipation, or diarrhea, and his weight had been stable.

Physical Examination.-This was an obese, pale man. The blood pressure was $130 / 80$, pulse 96 . On abdominal examination there was no tenderness.

Submitted for publication Aug. 12, 1959.

Markle Scholar (Dr. Starzl). Present address of Dr. Bernhard: Department of Surgery, Marquette University Medical School, Milwaukee.

From the Departments of Surgery, Northwestern University Medical School and the Veterans Administration Research Hospital, Chicago, and the University of Miami School of Medicine, Miami.
The liver was firm and palpable $5 \mathrm{~cm}$. below the right costal margin. No other abdominal masses were noted. The balance of the examination was normal, including esophagoscopy and sigmoidoscopy.

Laboratory Data.-Hemoglobin 7.8 gm., hematocrit $30 \%$, reticulocytes $2.1 \%$. Coagulogram was normal. Urinalysis, sulfobromophthalein retention, and Comb's test were normal. Stools were repeatedly guaiac-positive. Barium enema and upper gastrointestinal series were normal. Small bowel studies revealed irregularity of the mucosal pattern and an ulcerated filling defect in the fourth part of the duodenum near the ligament of Treitz (Fig. 1).

Fig. 1 (Case 1).-Preoperative gastrointestinal series. Arrows indicate tumor. Note ulceration in filling defect. Lesion was apparently at the duodenal-jejunal junction, but at surgery it was found to be on the duodenal side of the ligament of Treitz.

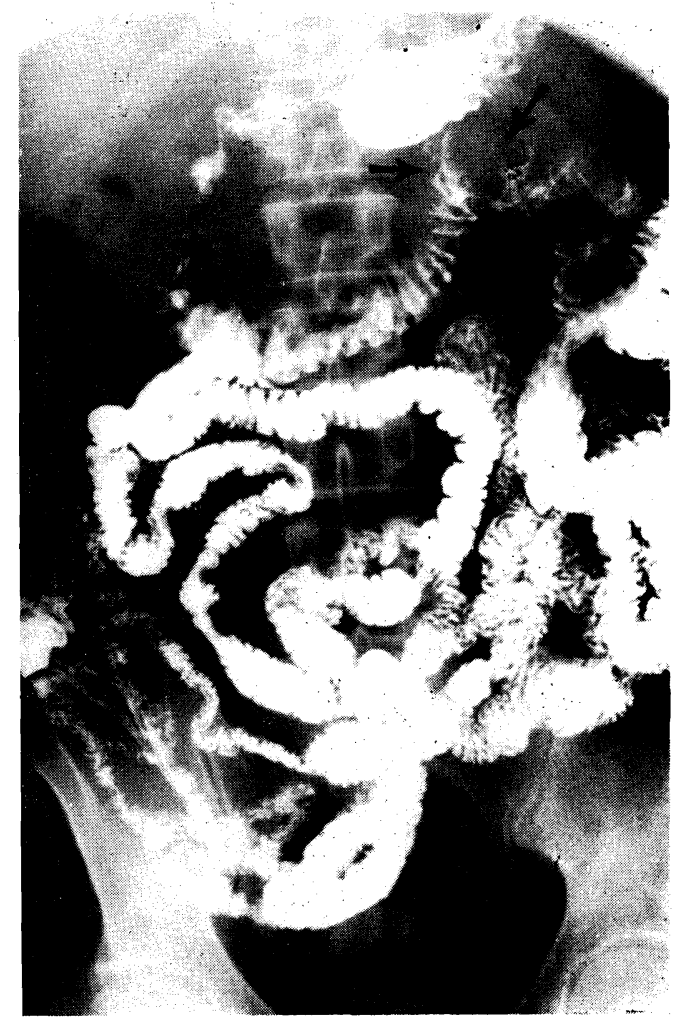




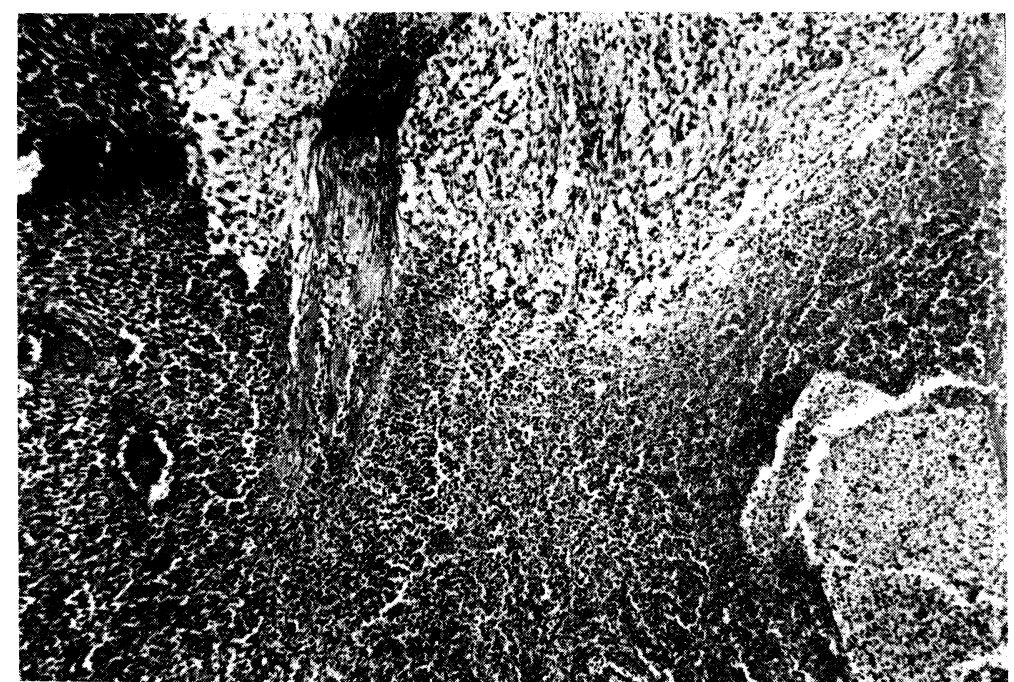

Fig. 2 (Case 1).-Microscopic appearance of tumor.
Course in Hospital: On Oct. 24, 1957, laparotomy was carried out through a left paramedian incision. A bluish-brown, irregular lime-sized tumor was in the wall of the duodenum at the duodenojejunal flexure projecting into the lumen and protruding through the serosa as well in dumbell fashion. The flexure was freed, and the third and fourth portions of the duodenum were resected with the proximal $10 \mathrm{~cm}$. of the jejunum. Bowel continuity was reestablished by an end-to-end anastomosis. Thrombophlebitis of the right calf developed postoperatively, and was treated with anticoagulants. The patient was discharged on Nov. 13, 1957, the 20th postoperative day.

Pathology.-The resected specimen contained a $4.5 \times 4 \times 3 \mathrm{~cm}$. transmural tumor. The serosa was intact over the mass. There was a central $1 \mathrm{~cm}$. area of ulceration on the mucosal surface. On cut section, the tumor was soft and grayish-yellow with central necrosis and hemorrhage. Microscopically the tumor showed no encapsulation and was made up of pleomorphic, atypical oval and spindle forms with hyperchromatic nuclei and occasional mitotic figures (Fig. 2). There were no lymph-node metastases. The diagnosis was leiomyosarcoma of the duodenum (Dr. J. J. Robinson). Confirmation of this opinion was obtained from the Armed Forces Institute of Pathology (No. 891532).

Follow-Up: Small bowel series one year after surgery was normal. $\mathrm{He}$ was last examined 18 months postoperatively and there was no evidence of recurrence.

Case 2 (Jackson Memorial Hospital, Miami). This 55-year-old white man was admitted with a four-month history of right upper quadrant pain and vomiting associated with a $25 \mathrm{lb}$. weight loss. The emesis and pain occurred concomitantly two or three hours after each meal. During this period starzl et al. he had two bouts of malodorous diarrhea, each lasting several days. There had been no jaundice.

Physical Examination.-This was a thin, chronically ill man. When the abdomen was examined, pain was engendered by deep palpatation in the right upper quadrant and there was a sense of resistance in this area. No definite mass could be defined. The balance of the examination was normal.

Fig. 3 (Case 2).-Gastrointestinal series. Arrows indicate obstructing mass.

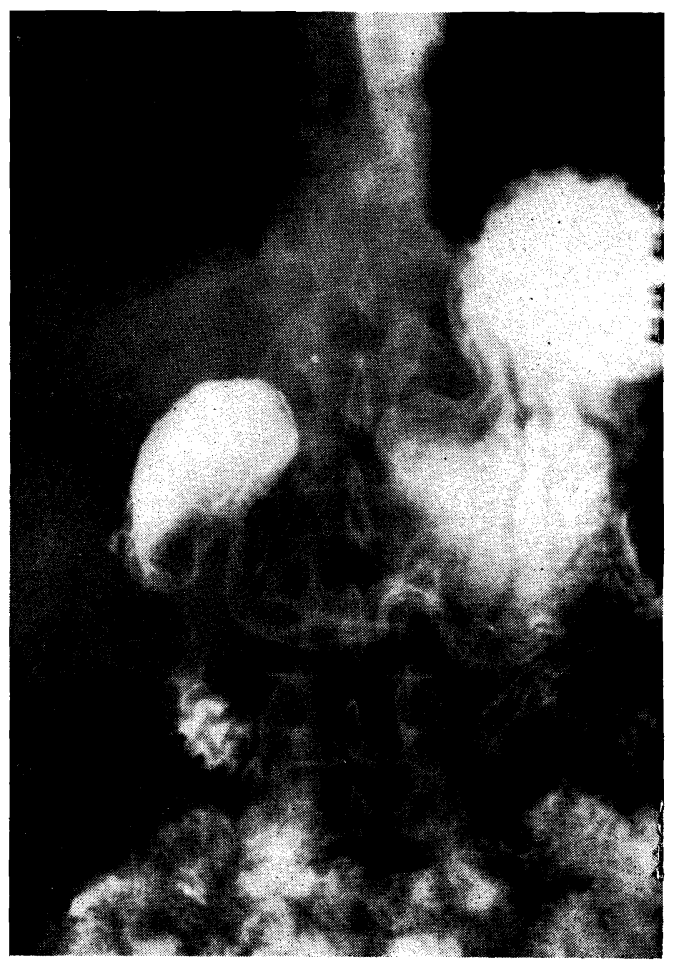

$185 / 529$ 


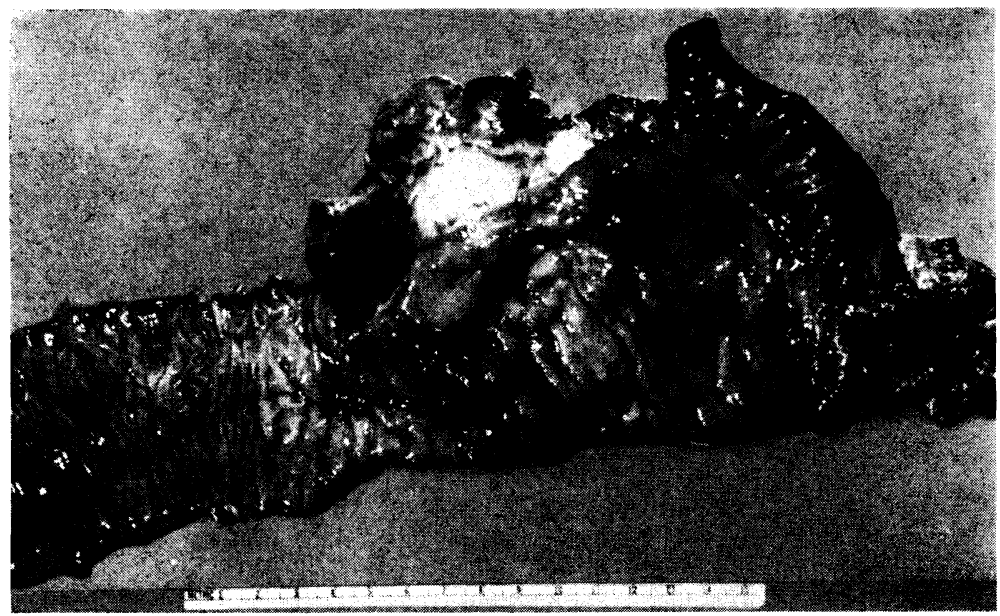

Fig. 4 (Case 2).-Gross specimen.

Laboratory Data.-Hemogram, urine, bilirubin, amylase, BUN, FBS, EKG, and chest $x$-ray were normal. Stool guaiac determinations were positive for occult blood. Cholecystogram and barium enema were normal. The gastrointestinal series revealed a long filling defect of the second part of the duodenum (Fig. 3), in which there was a feathered roughness of the medial duodenal wall. The first part of the duodenum was dilated (Fig. 3), secondary to the low-grade obstruction distally.

Course in Hospital.-The clinical impression was carcinoma of the pancreas. On Feb. 21, 1958, exploratory celiotomy was carried out, employing a midline incision with an oblique extension to the right. A $7 \mathrm{~cm}$. mass was found arising from the second part of the duodenum, invading the head of the pancreas. Edema extended from the tumor up to the gallbladder and through most of the supracolic space. The body and tail of the pancreas were firm but of normal size. The size of the common duct was normal, and the pancreatic duct could not be palpated. (Subsequently it was found to have a normal caliber.) Several enlarged nodes were found along the common duct and the left gastric artery, but these did not contain tumor on frozen section.

Pancreaticoduodenal resection was carried out, transecting the pancreas at the neck, removing $40 \%$ of the stomach, the entire duodenum, and the proximal jejunum. To obtain adequate margin
Fig. 5 (Case 2).High-power section of tumor.

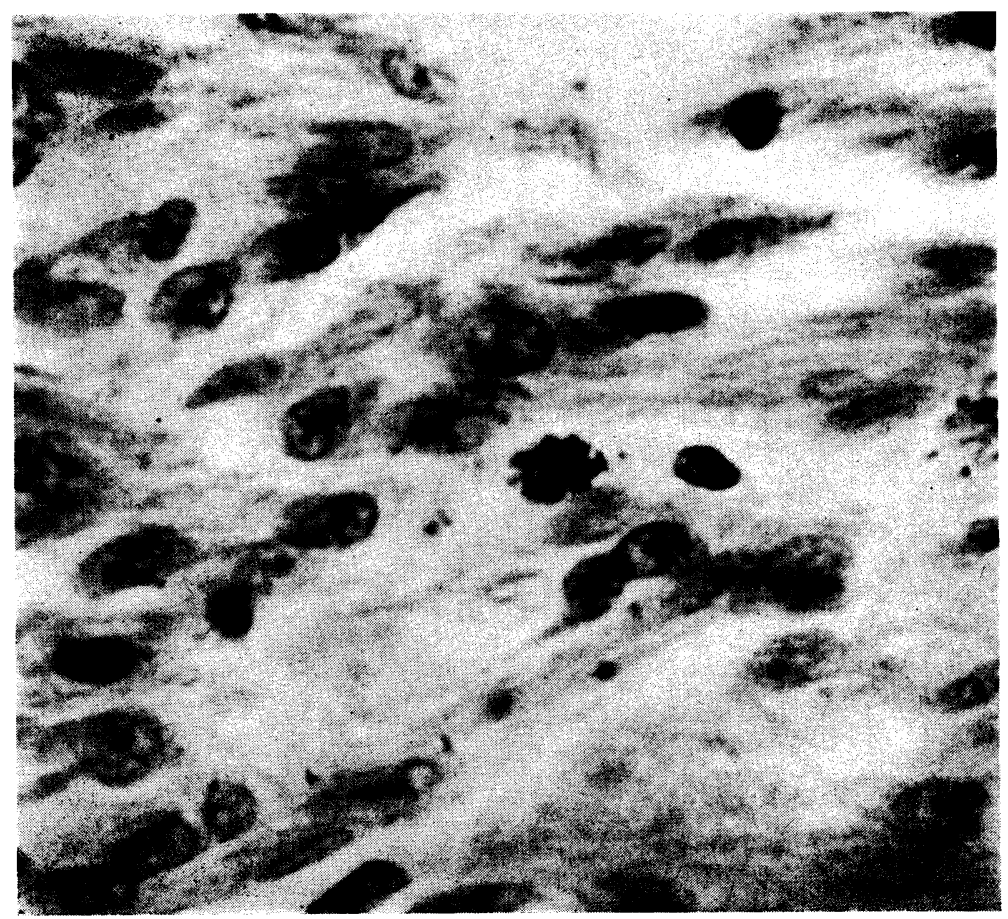

Vol. 80, Mar., 1960 
superiorly, the common duct was transected above the entrance of the cystic duct, necessitating cholecystectomy. Reconstruction was carried out with the method popularized by Cattell and Warren. ${ }^{2}$ There were no postoperative difficulties, and the patient was discharged from the hospital 11 days after surgery.

Pathology (Dr. Arthur C. Allen).-A $7 \times 6 \times 6$ $\mathrm{cm}$. mass (Fig. 4) originated from the second part of the duodenum, with extension into the head of the pancreas. The tumor was gray and firm upon transection. A section is illustrated which shows tumor structure at high power (Fig. 5). "There are whorled and intertwining bundles of neoplastic spindle cells, moderately pleomorphic nuclei, and occasional mitotic figures. The tumor has invaded pancreatic parenchyma." Diagnosis: leiomyosarcoma of duodenum.

Follow-Up.-During the first two months postoperatively the patient gained $25 \mathrm{lb}$. His health remained good until September, 1958, when he developed a marginal ulcer at the gastrojejunal stoma. Transabdominal vagotomy was performed (by Dr. Fernando Valverde) on Oct. 27, 1958. Complete abdominal exploration at this time revealed no evidence of recurrence. He was last seen 14 months postoperatively and was without evidence of recurrence.

\section{Comment}

Several reviews of leiomyosarcoma of the duodenum have been published in recent years., ${ }^{\mathbf{3}, \mathbf{6}}$ Common symptoms have been acute or chronic gastrointestinal hemorrhage, abdominal pain, weakness, weight loss, fever, vomiting, or the presence of a palpable mass. ${ }^{5}$ The proximity of lesions of the second part of the duodenum to the common duct would lead one to expect early jaundice, but this has not generally been the experience of others ${ }^{5}$ and was not the case in the presently reported tumor in this area.

There are insufficient data in the literature to allow an accurate opinion concerning the prognosis following resection of this rare duodenal malignancy. The longest reported survival after surgery was Martin's case ${ }^{3}$ in whom there was no evidence of recurrence after four years, but this is probably partly due to the submission of many case reports after a short postoperative period.
In the present study, the patients were well 14 and 18 months after surgery.

One of the patients in the present study is the 11th case in which pancreaticoduodenectomy was successfully carried out for duodenal leiomyosarcoma, ${ }^{5}$ the first report having been that of Shackelford. ${ }^{4}$ In most instances, and in this one as well, the tumor has been large, in some instances extraordinarily so. It has been pointed out by others ${ }^{1}$ that large size of pancreaticoduodenal tumors should not be a deterrent to an effort at resection if other factors are favorable, and this would seem to be a particularly applicable point of view in respect to leiomyosarcoma.

\section{Summary}

Leiomyosarcomata of the duodenum are rare, approximately 60 reports having been recorded in the world literature.

Two additional cases of this malignancy are documented, one in the second and the other in the fourth portion of the duodenum. The patients have been followed 14 and 18 months respectively after resection, with no evidence of recurrence.

The tumor in the second portion of the duodenum had invaded the pancreas and was removed with pancreaticoduodenal resection. The tumor in the fourth part of the duodenum was excised with segmental resection.

It is pointed out that the reported leiomyosarcomata of the second part of the duodenum have usually been large, and that size alone has not presented insurmountable difficulties in the performance of radical resection.

303 E. Chicago Ave. (11).

\section{REFERENCES}

1. Bowden, L.: Pancreatic Carcinoma: Disparity in Size of Neoplasm as Compared with Size of Gross Tumefaction, A.M.A. Arch. Surg. 76:559, 1958. 
2. Cattell, R. B., and Warren, K. W.: Surgery of the Pancreas, Philadelphia, W. B. Saunders Company, 1953.

3. Martin, J. F.: Leiomyosarcoma of the Small Intestine: A Report of 3 Cases and Review of the Literature, Am. J. Roentgenol. 74:1081, 1955.

4. Shackel ford, R. T.; Fisher, A. M., and Firor, W. B.: Duodenal Tumor of Unusual Character, Ann. Surg. $116: 864,1942$.
5. Starzl, T. E. ; Bernhard, V. M., and Heneger, G. C. : Leiomyosarcoma of the Duodenum: Collective Review, Surg. Gynec. \& Obst., to be published.

6. Weinstein, M., and Roberts, M.: Leiomyosarcoma of the Duodenum: Report of a Case and Summary of the Literature, A.M.A. Arch. Surg. $66: 318,1953$. 
